Pacific Journal of Mathematics

OPERATORS WHICH SATISFY POLYNOMIAL GROWTH 


\title{
OPERATORS WHICH SATISFY POLYNOMIAL GROWTH CONDITIONS
}

\author{
BRUCE A. BARNES
}

\begin{abstract}
Consider the class of bounded linear operators $S$ such that $\|\exp (i t S)\|$ has polynomial growth in $|t|$ on $\mathbf{R}$. In this paper it is shown that the operators in this class have many interesting properties in common with selfadjoint operators.
\end{abstract}

1. Introduction. If $S$ is a bounded linear selfadjoint operator on Hilbert space, then $\exp (i t S)$ is a unitary operator for all $t \in \mathbf{R}$, and thus

$$
\|\exp (i t S)\|=1 \quad(t \in \mathbf{R}) .
$$

When $S$ is an operator on a Banach space for which (1) holds, then $S$ is called Hermitian. The class of Hermitian operators has proved useful in the study of spectral operators. In this paper we study a more general class of operators, those for which the growth of $\|\exp (i t S)\|$ is at most polynomial in $t \in \mathbf{R}$, explicitly:

(2) $\exists K>0$ and $\exists \delta \geq 0$ such that $\|\exp (i t S)\| \leq K\left(1+|t|^{\delta}\right)$

Although this is a special class of operators, it does contain many interesting examples, and useful properties can be proved for operators in this class.

Throughout this paper $X$ is a Banach space. All operators on $X$ are automatically assumed to be linear and bounded. Let $\mathscr{P}(X)$ denote the set of all operators on $X$ for which (2) holds. Here is a list of some types of operators in $\mathscr{P}(X)$ :

A. Hermitian or Hermitian equivalent operators.

B. Operators on a Hilbert space of the form $T R S$ where $R \geq 0$ and $S T$ is selfadjoint.

C. Well-bounded operators ( $T$ is well-bounded means that for some interval $[a, b], \exists K>0$, such that for all polynomials $\left.p,\|p(T)\| \leq K\left(|p(b)|+\int_{a}^{b}\left|p^{\prime}(t)\right| d t\right)\right)$.

D. Nilpotent and projection operators. 
E. When $X$ is weakly complete, scalar-type spectral operators with real spectrum.

F. Algebraic operators with real spectrum.

G. Operators on Hilbert space which are in $G_{1}^{\text {loc }}$ and have real spectrum $\left(T \in G_{1}^{\text {loc }}\right.$ means that for some open neighborhood $U$ of $\sigma(T)$,

$$
\left.\left\|(\lambda-T)^{-1}\right\| \leq(\operatorname{dist}(\lambda, \sigma(T)))^{-1} \text { for all } \lambda \in U \backslash \sigma(T)\right) .
$$

That the operators which satisfy some property $(\mathrm{A})-(\mathrm{G})$ are in $\mathscr{P}(X)$ will be proved in $\S 2$.

What are the special properties of the operators in $\mathscr{P}(X)$ ? We prove that when $S \in \mathscr{P}(X)$, then

1. The spectrum of $S$ is real.

2. $\exists K>0$ and $\exists \delta>0$ such that for all $\lambda \in \mathbf{C}$ with $\operatorname{Im}(\lambda) \neq 0$,

$$
\left\|(\lambda-S)^{-1}\right\| \leq K\left(1+|\operatorname{Im}(\lambda)|^{-\delta}\right) .
$$

3. For all $\lambda \in \mathbf{C}, \lambda-S$ has finite ascent.

4. The closed subalgebra generated by $S$ and the identity is regular.

5. If the spectrum of $S$ contains more than one number, then $S$ has a proper closed hyper-invariant subspace.

Furthermore, we prove that when $S, T \in \mathscr{P}(X)$ and $S T=T S$, then $S+T \in \mathscr{P}(X)$ and $S T \in \mathscr{P}(X)$.

2. The class $\mathscr{P}(X)$. For an operator $S$, let $\mathscr{N}(S), \mathscr{R}(S), \alpha(S), \delta(S)$, and $\sigma(S)$ denote the null space of $S$, the range of $S$, the ascent of $S$, the descent of $S$, and the spectrum of $S$, respectively.

Consider the following three properties that may hold for an operator $S((\mathrm{II})$ is the defining condition for $S \in \mathscr{P}(X))$ :

I. $\exists K>0$ and $\exists \delta \geq 0$ such that $\|\exp (i n S)\| \leq K\left(1+|n|^{\delta}\right)(n \in \mathbf{Z})$;

II. $\exists K>0$ and $\exists \delta \geq 0$ such that $\|\exp (i t S)\| \leq K\left(1+|t|^{\delta}\right)(t \in \mathbf{R})$;

III. $\sigma(S) \subseteq \mathbf{R}$ and $\exists K>0$ and $\exists \delta>0$ such that when $\lambda \in \mathbf{C}$ with $\operatorname{Im}(\lambda) \neq 0$, then $\left\|(\lambda-S)^{-1}\right\| \leq K\left(1+|\operatorname{Im}(\lambda)|^{-\delta}\right)$.

In fact these three conditions are equivalent (the values of $K$ and $\delta$ may differ in the different conditions). The equivalence of (I) and (II) is an elementary fact. For suppose (I) holds for $S$, and $K$ and $\delta$ are as in (I). Since $t \rightarrow\|\exp (i t S)\|$ is continuous, $\exists J>0$ such that $\sup \{\|\exp (i t S)\|: t \in[-1,1]\} \leq J$. Then for $t \in \mathbf{R}, \exists v \in(-1,1)$ and $n \in \mathbf{Z}$ such that $t=v+n$ and $|n| \leq|t|$. Thus

$\|\exp (i t S)\| \leq\|\exp (i v S)\|\|\exp (i n S)\| \leq J K\left(1+|n|^{\delta}\right) \leq J K\left(1+|t|^{\delta}\right)$. 
From this it is clear that (I) and (II) are equivalent.

On the way to proving the equivalence of (I)-(III) we establish several important results.

Theorem 1. Assume (II) holds for an operator S. Fix $\lambda \in \mathbf{C}$ with $c=\operatorname{Im}(\lambda) \neq 0$. If $c>0$, then

$$
(\lambda-S)^{-1}=-i \int_{0}^{\infty} e^{i \lambda t} e^{-i t S} d t
$$

If $c<0$, then

$$
(\lambda-S)^{-1}=i \int_{-\infty}^{0} e^{i \lambda t} e^{-i t S} d t
$$

Proof. We prove the formula in the case $c>0$; the proof of the other case is similar. For $w>0$,

$$
i(\lambda-S) \int_{0}^{w} e^{i(\lambda-S) t} d t=\int_{0}^{w}\left[\frac{d}{d t}\left(e^{i(\lambda-S) t}\right)\right] d t=e^{i(\lambda-S) w}-I .
$$

Also, $\left\|e^{i(\lambda-S) w}\right\|=e^{-c w}\left\|e^{-i w S}\right\| \leq e^{-c w} K\left(1+w^{\delta}\right)$. Thus $\left\|e^{l(\lambda-S) w}\right\| \rightarrow$ 0 as $w \rightarrow \infty$. This proves

$$
i(\lambda-S) \int_{0}^{\infty} e^{i(\lambda-S) t} d t=-I
$$

Corollary 2. (II) $\Rightarrow$ (III).

Proof. Assume (II) holds. Assume $\lambda \in \mathbf{C}$ with $c=\operatorname{Im}(\lambda) \neq 0$. We assume $c>0$. Then by Theorem 1

$$
(\lambda-S)^{-1}=-i \int_{0}^{\infty} e^{i \lambda t} e^{-i S t} d t
$$

Thus,

$$
\left\|(\lambda-S)^{-1}\right\| \leq \int_{0}^{\infty}\left\|e^{i(\lambda-S) t}\right\| d t
$$

Now

$$
\left\|e^{i(\lambda-S) t}\right\| \leq e^{-c t} K\left(1+|t|^{\delta}\right) .
$$

The definite integrals involved are evaluated by

$$
\int_{0}^{\infty} t^{\delta} e^{-c t} d t=\Gamma(\delta+1) c^{-(\delta+1)}
$$

where $\Gamma$ is the gamma-function. Thus (III) holds for the appropriate choice of constants. 
THEOREM 3. (III) $\Rightarrow$ (II).

Proof. Assume $S$ is an operator for which (III) holds. We may assume $\|S\| \leq 1$, so $\sigma(S) \subseteq[-1,1]$. Fix $\varepsilon, 0<\varepsilon \leq 1$. Define paths $\gamma_{j}$, $1 \leq j \leq 4$, by

$$
\left.\begin{array}{c}
\gamma_{1}(t)=(2-4 t)+i \varepsilon, \\
\gamma_{3}(t)=(-2+4 t)-i \varepsilon,
\end{array}\right\} \quad t \in[0,1],
$$

Let $\gamma$ be the closed path encircling $\sigma(S)$ counter-clockwise defined by $\gamma=\gamma_{1}+\gamma_{2}+\gamma_{3}+\gamma_{4}$. By the holomorphic operational calculus we have

$$
e^{i t S}=\frac{1}{2 \pi i} \int_{\gamma} e^{i t \lambda}(\lambda-S)^{-1} d \lambda \quad(t \in \mathbf{R}) .
$$

We show (II) holds by making estimates on

$$
\left\|\int_{\gamma_{j}} e^{i t \lambda}(\lambda-S)^{-1} d \lambda\right\|, \quad 1 \leq j \leq 4 .
$$

We make the estimates for $j=1,2$; the computations for $j=3,4$ are similar.

$$
\begin{aligned}
\left\|\int_{\gamma_{1}} e^{i t \lambda}(\lambda-S)^{-1} d \lambda\right\| & \leq \int_{0}^{1}\left|e^{i t \gamma_{1}(x)}\right|\left\|\left(\gamma_{1}(x)-S\right)^{-1}\right\|\left|\gamma_{1}^{\prime}(x)\right| d x \\
& \leq 4 \int_{0}^{1} e^{-\varepsilon t} K\left(1+\varepsilon^{-\delta}\right) d x=4 K e^{-\varepsilon t}\left(1+\varepsilon^{-\delta}\right) .
\end{aligned}
$$

Next, let

$$
J=\sup \left\{\left\|((-2+i x)-S)^{-1}\right\|: x \in \mathbf{R}\right\} .
$$

Note that $J$ is finite. Then for $t \neq 0$

$$
\begin{aligned}
\left\|\int_{\gamma_{2}} e^{i t \lambda}(\lambda-S)^{-1} d \lambda\right\| & \leq \int_{-\varepsilon}^{\varepsilon}\left|e^{i t \gamma_{2}(x)}\right|\left\|\left(\gamma_{2}(x)-S\right)^{-1}\right\| d x \\
& \leq \int_{-\varepsilon}^{\varepsilon} e^{t x} J d x=J t^{-1}\left(e^{\varepsilon t}-e^{-\varepsilon t}\right) .
\end{aligned}
$$

Similar estimates hold for the norm of the path integrals:

$$
\begin{aligned}
& \left\|\int_{\gamma_{3}} e^{i t \lambda}(\lambda-S)^{-1} d \lambda\right\| \leq 4 K e^{\varepsilon t}\left(1+\varepsilon^{-\delta}\right), \\
& \left\|\int_{\gamma_{4}} e^{i t \lambda}(\lambda-S)^{-1} d \lambda\right\| \leq M t^{-1}\left(e^{\varepsilon t}-e^{-\varepsilon t}\right)
\end{aligned}
$$


where $t \neq 0$ and

$$
M=\sup \left\{\left\|((2+i x)-S)^{-1}\right\|: x \in \mathbf{R}\right\} .
$$

Assuming $|t| \geq 1$, let $\varepsilon=|t|^{-1}$ in the estimates above. This gives for $|t| \geq 1,\|\exp (i t S)\| \leq K^{\prime}\left(1+|t|^{\delta}\right)$ for some choice of $K^{\prime}$. Thus, (II) holds.

REMARK. It is useful to note that (III) is true if $\sigma(S) \subseteq \mathbf{R}$ and we assume only that the inequality in (III) holds for all $\lambda \in U, \operatorname{Im}(\lambda) \neq 0$, where $U$ is some open neighborhood of $\sigma(S)$. For it is well known that $\lim _{|\lambda| \rightarrow \infty}\left\|(\lambda-S)^{-1}\right\|=0$. Therefore $\exists J>0$ such that $\left\|(\lambda-S)^{-1}\right\| \leq J$ for $\lambda \notin U$. Then for $\lambda \in \mathbf{C}, \operatorname{Im}(\lambda) \neq 0$,

$$
\left\|(\lambda-S)^{-1}\right\| \leq(J+K)\left(1+|\operatorname{Im}(\lambda)|^{-\delta}\right) .
$$

LemMA 4. If $S \in \mathscr{P}(X)$, then $S^{2} \in \mathscr{P}(X)$.

Proof. We may assume $\|S\| \leq 1$. Fix $\varepsilon, 0<\varepsilon \leq 1$. Define the paths $\gamma_{j}, 1 \leq j \leq 4$, and $\gamma$, just as in the proof of Theorem 3 . Then

$$
\exp \left(i t S^{2}\right)=\frac{1}{2 \pi i} \int_{\gamma} e^{i t \lambda^{2}}(\lambda-S)^{-1} d \lambda \quad(t \in \mathbf{R}) .
$$

For $1 \leq j \leq 4$, let

$$
A_{j}=\left\|\int_{\gamma_{j}} e^{i t \lambda^{2}}(\lambda-S)^{-1} d \lambda\right\| .
$$

By Corollary $2 \exists K \geq 0$ and $\exists \delta \geq 0$ such that

$$
\left\|(\lambda-S)^{-1}\right\| \leq K\left(1+|\operatorname{Im}(\lambda)|^{-\delta}\right)
$$

whenever $\operatorname{Im}(\lambda) \neq 0$. The following estimates hold (the argument being similar to the proof of Theorem 3): For $t \neq 0$,

$$
\begin{aligned}
& A_{1} \leq(2 \varepsilon t)^{-1}\left(e^{4 t \varepsilon}-e^{-4 t \varepsilon}\right) K\left(1+\varepsilon^{-\delta}\right) \\
& A_{2} \leq J(4 t)^{-1}\left(e^{4 t \varepsilon}-e^{-4 t \varepsilon}\right) \\
& A_{3} \leq(2 \varepsilon t)^{-1}\left(e^{4 t \varepsilon}-e^{-4 t \varepsilon}\right) K\left(1+\varepsilon^{-\delta}\right) \\
& A_{4} \leq M(4 t)^{-1}\left(e^{4 t \varepsilon}-e^{-4 t \varepsilon}\right)
\end{aligned}
$$

Here $M>0$ and $J>0$ are fixed constants. Then letting $\varepsilon=|t|^{-1}$ when $|t| \geq 1$, we have that $\left\|\exp \left(i t S^{2}\right)\right\|$ is polynomial in $|t|$.

THEOREM 5.

(1) If $T, S \in \mathscr{P}(X)$ and $S T=T S$, then $S+T \in \mathscr{P}(X)$; 
(2) If $T, S \in \mathscr{P}(X)$ and $S T=T S$, then $S T \in \mathscr{P}(X)$;

(3) If $S \in \mathscr{P}(X)$ and $p(\lambda)$ is a polynomial with coefficients in $\mathbf{R}$, then $p(S) \in \mathscr{P}(X)$.

Proof. (1) is easily proved and (3) follows from (1) and (2). To prove (2) suppose $S$ and $T$ are as in the statement of (2). Then

$$
S T=\frac{1}{2}\left\{(S+T)^{2}-S^{2}-T^{2}\right\} .
$$

By Lemma $4,(S+T)^{2}, S^{2}$, and $T^{2}$ are in $\mathscr{P}(X)$. It follows that $S T \in$ $\mathscr{P}(X)$.

The algebraic closure properties of the class $\mathscr{P}(X)$ proved in Theorem 5 contrast with the failure of these properties relative to interesting subclasses of $\mathscr{P}(X)$. In particular:

(1) The square of an Hermitian operator need not be Hermitian [2, Example 4.13, p. 107].

(2) The sum of commuting scalar-type spectral operators need not be of scalar type [2, Chapter 9].

(3) The sum and product of commuting well-bounded operators need not be well-bounded [2, p. 362].

There is another class of operators defined in terms of a growth condition of the resolvent operator which is of interest here. Define an operator $S$ to be in $\mathscr{G}(X)$ when

$$
\exists K>0 \text { and } \exists \delta>0 \text { such that }\left\|(\lambda-S)^{-1}\right\| \leq K\left(1+d(\lambda)^{-\delta}\right)
$$

whenever $\lambda \notin \sigma(S)$; here $d(\lambda)$ is the distance from $\lambda$ to $\sigma(S)$.

Just as in the Remark following Theorem 3, we note that the inequality in the defining property for $\mathscr{G}(X)$ need only be assumed to hold for all $\lambda \in U, \lambda \notin \sigma(S)$, where $U$ is some open neighborhood of $\sigma(S)$.

We have from Corollary 2 that (II) $\Rightarrow$ (III) and this gives immediately the following result.

Proposition 6. If $S \in \mathscr{G}(X)$ and $\sigma(S) \subseteq \mathbf{R}$, then $S \in \mathscr{P}(X)$.

Next we verify that the examples of types of operators listed in the Introduction are in $\mathscr{P}(X)$.

THEOREM 7. If $S$ is an operator with one of the properties $(\mathrm{A})-(\mathrm{G})$, then $S \in \mathscr{P}(X)$.

Proof. (A): If $S$ is Hermitian or Hermitian equivalent, then $S$ satisfies (II) with $\delta=0$ by [2, Theorem 4.7, p. 104] and [2, Definition 4.16 , p. 108]. 
(B): Assume $W$ has the form $W=T R S$ as described in (B). Then by [1, Theorem 3.4] $\exists K>0$ such that

$$
\|\exp (i t W)\| \leq K(1+|t|) \quad(t \in \mathbf{R}) .
$$

(C): Assume $S$ is a well-bounded operator on $X$. Let $[a, b]$ be the given interval in the definition; see [2, Def. 15.1, p. 287] where $J=$ $[a, b]$. When $f(x)$ is absolutely continuous on $[a, b]$, let

$$
\||| f|||=| f(b)\left|+\int_{a}^{b}\right| f^{\prime}(x) \mid d x
$$

as in [2, p. 287]. By [2, Lemma 15.2, p. 287] $\exists K>0$ such that

$$
\|\exp (i t S)\| \leq K\left\|\left|e^{i t x}\right|\right\| \quad(t \in \mathbf{R}) .
$$

Since $\left\|\left|e^{i t x}\right|\right\|=1+|t|(b-a), S$ satisfies (II).

(D): This is an easy computation. For example, if $P^{2}=P$, then

$$
\exp (i t P)=e^{i t} P+(I-P) .
$$

Thus in this case $\exists K>0$ such that

$$
\|\exp (i t P)\| \leq K \quad(t \in \mathbf{R}) .
$$

(E): Assume $X$ is weakly complete and that $S$ is a scalar-type spectral operator on $X$ with $\sigma(S) \subseteq \mathbf{R}$. By [2, Theorem 6.13, p. 166] $\exists M>0$ such that for each rational function $g$ with poles outside of $\sigma(S)$

$$
\|g(S)\| \leq M \sup \{|g(z)|: z \in \sigma(S)\} .
$$

Fix $\lambda \notin \sigma(S)$, and let $g(z)=(\lambda-z)$. By the inequality above

$$
\left\|(\lambda-S)^{-1}\right\| \leq M \sup \left\{|\lambda-z|^{-1}: z \in \sigma(S)\right\}=M d(\lambda)^{-1} .
$$

Thus $S \in \mathscr{G}(X)$ in this case.

(F): Assume $S$ is an algebraic operator with $\sigma(S) \subseteq \mathbf{R}$. Then by [5, p. 338] $S$ has the form

$$
S=\sum_{k=1}^{m} \lambda_{k} E_{k}+N
$$

where $E_{k} E_{j}=\delta_{k, j} E_{k}, 1 \leq k, j \leq m,\left\{\lambda_{1}, \ldots, \lambda_{m}\right\} \subseteq \mathbf{R}$, and $N$ is nilpotent with $N E_{k}=E_{k} N$ for all $k$. Now as we have noted, $E_{k} \in$ $\mathscr{P}(X)$ for all $k$ and $N \in \mathscr{P}(X)$. It follows from Theorem 5 that $S \in \mathscr{P}(X)$.

(G): Let $S$ be an operator on Hilbert space, $S \in G_{1}^{\text {loc }}$, and with $\sigma(S) \subseteq \mathbf{R}$. Since $S \in G_{1}^{\text {loc }}$, there $\exists U$ an open neighborhood of $\sigma(S)$ 
such that $\left\|(\lambda-S)^{-1}\right\| \leq d(\lambda)^{-1}$ for all $\lambda \in U, \lambda \notin \sigma(S)$ [3, Definition 7.3.17, p. 294]. Therefore $S \in \mathscr{G}(X)$ in this case.

REMARK. Assume $T$ is an invertible operator and $\exists K>0$ and $\exists \delta \geq 0$ such that

$$
\left\|T^{n}\right\| \leq K\left(1+|n|^{\delta}\right) \quad(n \in \mathbf{Z}) .
$$

Then $\sigma(T) \subseteq\{\lambda:|\lambda|=1\}$. Suppose this inclusion is proper. Then $\exists S$ an operator such that $T=e^{i S}$. Thus, by the inequality for $\left\|T^{n}\right\|, S$ satisfies (I), so $S \in \mathscr{P}(X)$.

3. Properties of operators in $\mathscr{P}(X)$. If $S$ is a selfadjoint operator on Hilbert space, then for $\lambda \in \mathbf{C}, \mathscr{N}\left((\lambda-S)^{2}\right)=\mathscr{N}(\lambda-S)$. Thus in this case $\alpha(\lambda-S)$ is always either 0 or 1 . Also, if $(\lambda-S)$ has closed range and $\lambda \in \sigma(S)$, then $\lambda$ is an isolated point of $\sigma(S)$ and a pole of the resolvent operator. Operators in $\mathscr{P}(X)$ have similar properties which we elucidate in the first part of this section. If $\delta \in \mathbf{R}$, then let $[\delta]$ denote the smallest integer $n$ with $\delta \leq n$.

Theorem 8. Assume $S \in \mathscr{P}(X)$. Then $\exists m \in \mathbf{Z}, m \geq 0$, such that $\alpha(\lambda-S) \leq m$ for all $\lambda \in \mathbf{C}$.

Proof. We may assume $\lambda \in \sigma(S)$, and in fact, we may assume that $\lambda=0$ (since we may replace $S$ in the following proof by $\lambda-S$ ). We prove $\alpha(S)$ is finite. By Corollary $2 \exists K>0$ and $\delta>0$ such that

$$
\left\|(i t-S)^{-1}\right\| \leq K\left(1+|t|^{-\delta}\right) \quad(t \in \mathbf{R}, t \neq 0) .
$$

Let $m=[\delta]+1$. Then

$$
\lim _{t \rightarrow 0^{+}}(i t)^{m}(i t-S)^{-1}=0 .
$$

Suppose $\alpha(S)>m$. Then we can choose $x \in X$ and $\beta \in X^{\prime}$ such that $S^{m+1}(x)=0, S^{m}(x) \neq 0$, and $\beta\left(S^{m} x\right)=1$. Define a continuous linear functional $\varphi$ on the space of bounded operators by $\varphi(T)=\beta(T x)$. By Theorem 1,

$$
(i t-S)^{-1}=-i \int_{0}^{\infty} e^{-t x} e^{-i x S} d x \quad(t>0) .
$$

Then for $t>0$

$$
\varphi\left((i t-S)^{-1}\right)=-i \int_{0}^{\infty} e^{-t x}\left(\sum_{k=0}^{\infty} \frac{(-i x)^{k}}{k !} \varphi\left(S^{k}\right)\right) d x
$$


Now $\varphi\left(S^{k}\right)=\beta\left(S^{k} x\right)=0$ for $k>m$, so for $t>0$

$$
\begin{aligned}
&(i t)^{m} \varphi\left((i t-S)^{-1}\right)=-(i)^{m+1} t^{m} \sum_{k=0}^{m} \frac{(-i)^{k}}{k !}\left[\int_{0}^{\infty} x^{k} e^{-t x} d x\right] \varphi\left(S^{k}\right) \\
&=-(i)^{m+1} t^{m} \sum_{k=0}^{m} \frac{(-i)^{k}}{k !}\left[\frac{(k+1) !}{t^{k+1}}\right] \varphi\left(S^{k}\right) \\
&=-(i)^{2 m+1}(m+1) t^{-1}+\{\text { terms involving nonnegative } \\
&\text { powers of } t\} .
\end{aligned}
$$

Thus $(i t)^{m} \varphi\left((i t-S)^{-1}\right) \nrightarrow 0$ as $t \rightarrow 0^{+}$, a contradiction. We conclude that $\alpha(S) \leq m$.

TheOREM 9. Assume $S \in \mathscr{P}(X)$. There exists an integer $m \geq 0$ such that for all $\lambda \in \mathbf{C}$

$$
\mathscr{R}\left((\lambda-S)^{j}\right)^{-}=\mathscr{R}\left((\lambda-S)^{m}\right)^{-} \text {for } j \geq m .
$$

In particular, if $\mathscr{R}(\lambda-S)$ is closed, then $\delta(\lambda-S) \leq m$. In this case if $\lambda \in \sigma(S)$, then $\lambda$ is an isolated point of $\sigma(S)$ and a pole of the resolvent operator.

Proof. Fix $\lambda \in \mathbf{C}$. Now $S^{\prime} \in \mathscr{P}\left(X^{\prime}\right)$, so by Theorem $8 \exists$ a nonnegative integer $m$ such that $\alpha\left(\lambda-S^{\prime}\right) \leq m$. Thus, $\mathscr{N}\left(\left(\lambda-S^{\prime}\right)^{j}\right)=\mathscr{N}\left(\left(\lambda-S^{\prime}\right)^{m}\right)$ for $j \geq m$. It follows that $\mathscr{R}\left((\lambda-S)^{j}\right)^{-}=\mathscr{R}\left((\lambda-S)^{m}\right)^{-}$for $j \geq m$.

Now suppose $\mathscr{R}(\lambda-S)$ is closed. Then $\mathscr{R}\left((\lambda-S)^{j}\right)$ is closed for all $j \geq 1$. Thus by what was proved above $\mathscr{R}\left((\lambda-S)^{j}\right)=\mathscr{R}\left((\lambda-S)^{m}\right)$ for $j \geq m$. This proves $\delta(\lambda-S) \leq m$. Assume $\lambda \in \sigma(S)$. We have that both $\alpha(\lambda-S)$ and $\delta(\lambda-S)$ are finite. It follows from this that $\lambda$ is an isolated point of $\sigma(S)$ and $\lambda$ is a pole of the resolvent operator; see [5, Theorem 10.2, p. 330].

When $S \in \mathscr{G}(X)$, then $S$ has the strong property that any isolated point in $\sigma(S)$ is a pole of the resolvent. This is an easy fact which we prove now.

Proposition 10. If $S \in \mathscr{G}(X)$ and $\lambda_{0}$ is an isolated point of $\sigma(S)$, then $\lambda_{0}$ is a pole of the resolvent.

Proof. Let $U$ be an open neighborhood of $\lambda_{0}$ with $\sigma(S) \cap U=\left\{\lambda_{0}\right\}$. Let

$$
\gamma(t)=\lambda_{0}+r e^{i t}, \quad t \in[0,2 \pi]
$$


where $r>0$ is chosen so that $\gamma(t) \in U$ for all $t$. Since $S \in \mathscr{G}(X)$, $\exists K>0$ and $\exists \delta>0$ such that for $\lambda \notin \sigma(S)$

$$
\left\|(\lambda-S)^{-1}\right\| \leq K\left(1+d(\lambda)^{-\delta}\right) .
$$

Let $m=[\delta]+1$. Then

$$
\begin{aligned}
\left\|\int_{\gamma}\left(\lambda-\lambda_{0}\right)^{m}(\lambda-S)^{-1} d \lambda\right\| & \leq \int_{0}^{2 \pi} r^{m} K\left(1+r^{-m}\right) r d t \\
& =2 \pi K\left(r^{m+1}+r\right) .
\end{aligned}
$$

Now let $r \rightarrow 0^{+}$. This proves $\lambda_{0}$ is a pole of the resolvent by [5, pp. 328-329] (in the notation in [5], we have proved $B_{n}=0$ for $n \geq m+1$ ).

Now we consider other properties of selfadjoint operators on a Hilbert space which hold for operators in $\mathscr{P}(X)$. When $S$ is selfadjoint, then the closed subalgebra generated by $S$ and the identity operator can be identified with $C(\Omega)$, the algebra of all complex-valued continuous functions on a compact set $\Omega$. The algebra $C(\Omega)$ is regular in the sense that if $\Gamma$ is a closed subset of $\Omega$ and $\omega \in \Omega \backslash \Gamma$, then there is a function $f \in C(\Omega)$ such that $f(\Gamma)=\{0\}$ and $f(\omega) \neq 0$. Now assume $S \in \mathscr{P}(X)$. Denote by $A[S]$ the closed subalgebra generated by $S$ and the identity operator. Via standard Gelfand theory, the Banach algebra $A[S]$ is identified with some subalgebra $\mathscr{A}$ of $C(\Omega)$. Then $A[S]$ is regular if whenever $\Gamma$ is a closed subset of $\Omega$ and $\omega \in \Omega \backslash \Gamma$, then there is a function $f \in \mathscr{A}$ such that $f(\Gamma)=\{0\}$ and $f(\omega) \neq 0$. We note below that $A[S]$ is regular.

TheOREM 13. Assume $S \in \mathscr{P}(K)$. Then

1. $A[S]$ is regular; and

2. if $\sigma(S)$ contains more than one point, then $S$ has a closed proper hyper-invariant subspace.

A proof of Theorem 13 can be constructed along the same lines as the proof of Theorem 5.2 in [1]. We give a brief indication of what is involved in such a proof. The key condition is that $\exists K>0$ and $\exists \delta>0$ with

$$
\|\exp (i n S)\| \leq K\left(1+|n|^{\delta}\right) \quad(n \in \mathbf{Z}) .
$$

Let $\alpha_{n}=\max (\|\exp (i n S)\|,\|\exp (-i n S)\|)$ for $n \in \mathbf{Z}$, and set $\alpha=\left\{\alpha_{n}\right\}$. The space of complex sequences $b=\left\{b_{k}\right\}_{k \in Z}$ with the property

$$
\|b\|=\sum_{k \in \mathbf{Z}}\left|b_{k}\right| \alpha_{k}<\infty
$$


is a commutative convolution Banach algebra; see [3, pp. 118-120]. Denote this Banach algebra by $W(\alpha)$. Now $W(\alpha)$ is semisimple (being a subalgebra of $\left.l^{1}(Z)\right)$ and regular by [3,pp. 214-215]. The conclusion that $W(\alpha)$ is regular uses the key condition above. Define an algebra homomorphism $\varphi: W(\alpha) \rightarrow A[S]$ by

$$
\varphi\left(\left\{b_{k}\right\}\right)=\sum_{k=-\infty}^{\infty} b_{k} \exp (i k S) .
$$

We may assume $\|S\| \leq 1$, in which case the subalgebra $\left\{\varphi\left(\left\{a_{k}\right\}\right):\left\{a_{k}\right\}\right.$ $\in W(\alpha)\}$ strongly separates points of the Gelfand space of $A[S]$. This is enough to conclude that the results in Theorem 13 hold by using [1, Theorem 5.1].

After the completion of this paper, the author found a recent paper by $\mathrm{T}$. Pytlik which contains results related to some of the results given in §3: Analytic semigroups in Banach algebras and a theorem of Hille, Colloq. Math. 51 (1987), 287-293.

\section{REFERENCES}

[1] B. A. Barnes, Operators symmetric with respect to a pre-innerproduct; recently submitted.

[2] H. R. Dowson, Spectral Theory of Linear Operators, Academic Press, LondonNew York-San Franciso, 1978.

[3] I. Gelfand, D. Raikov, and G. Shilov, Commutative Normed Rings, Chelsea, New York, 1964.

[4] V. I. Istratescu, Introduction to Linear Operator Theory, Marcel Dekker, Inc., New York-Basel, 1981.

[5] D. Lay and A. E. Taylor, Introduction to Functional Analysis, John Wiley \& Sons, New York-Chichester-Brisbane-Toronto, 1980.

Received January 8, 1988 and in revised form April 22, 1988.

UNIVERSITY OF OREGON

EUGENE, OR 97403 



\section{PACIFIC JOURNAL OF MATHEMATICS EDITORS}

\author{
V. S. VARADARAJAN \\ (Managing Editor) \\ University of California \\ Los Angeles, CA 90024 \\ Herbert Clemens \\ University of Utah \\ Salt Lake City, UT 84112 \\ THOMAs ENRIGHT \\ University of California, San Diego \\ La Jolla, CA 92093
}

R. FINN

Stanford University

Stanford, CA 94305

HeRmann FlaschKa

University of Arizona

Tucson, AZ 85721

VAUGHAN F. R. JONES

University of California

Berkeley, CA 94720

Steven KerckhofF

Stanford University

Stanford, CA 94305
ROBION KIRBY

University of California

Berkeley, CA 94720

C. C. MOORE

University of California

Berkeley, CA 94720

HAROLD STARK

University of California, San Diego

La Jolla, CA 92093

\section{ASSOCIATE EDITORS}
R. ARENS
E. F. BECKENBACH
B. H. NEUMANN
F. Wolf
K. YoshidA
(1906-1982)

\section{SUPPORTING INSTITUTIONS}

UNIVERSITY OF ARIZONA

UNIVERSITY OF BRITISH COLUMBIA

CALIFORNIA INSTITUTE OF TECHNOLOGY

UNIVERSITY OF CALIFORNIA

MONTANA STATE UNIVERSITY

UNIVERSITY OF NEVADA, RENO

NEW MEXICO STATE UNIVERSITY OREGON STATE UNIVERSITY

\author{
UNIVERSITY OF OREGON \\ UNIVERSITY OF SOUTHERN CALIFORNIA \\ STANFORD UNIVERSITY \\ UNIVERSITY OF HAWAII \\ UNIVERSITY OF TOKYO \\ UNIVERSITY OF UTAH \\ WASHINGTON STATE UNIVERSITY \\ UNIVERSITY OF WASHINGTON
}

The Supporting Institutions listed above contribute to the cost of publication of this Journal, but they are not owners or publishers and have no responsibility for its content or policies.

Mathematical papers intended for publication in the Pacific Journal of Mathematics should be in typed form or offset-reproduced (not dittoed), double spaced with large margins. Please do not use built up fractions in the text of the manuscript. However, you may use them in the displayed equations. Underline Greek letters in red, German in green, and script in blue. The first paragraph must be capable of being used separately as a synopsis of the entire paper. In particular it should contain no bibliographic references. Please propose a heading for the odd numbered pages of less than 35 characters. Manuscripts, in triplicate, may be sent to any one of the editors. Please classify according to the scheme of Math. Reviews, Index to Vol. 39. Supply name and address of author to whom proofs should be sent. All other communications should be addressed to the managing editor, or Elaine Barth, University of California, Los Angeles, California 90024.

There are page-charges associated with articles appearing in the Pacific Journal of Mathematics. These charges are expected to be paid by the author's University, Government Agency or Company. If the author or authors do not have access to such Institutional support these charges are waived. Single authors will receive 50 free reprints; joint authors will receive a total of 100 free reprints. Additional copies may be obtained at cost in multiples of 50 .

The Pacific Journal of Mathematics is issued monthly as of January 1966. Regular subscription rate: $\$ 190.00$ a year (5 Vols., 10 issues). Special rate: $\$ 95.00$ a year to individual members of supporting institutions.

Subscriptions, orders for numbers issued in the last three calendar years, and changes of address should be sent to Pacific Journal of Mathematics, P.O. Box 969, Carmel Valley, CA 93924, U.S.A. Old back numbers obtainable from Kraus Periodicals Co., Route 100, Millwood, NY 10546.

The Pacific Journal of Mathematics at P.O. Box 969, Carmel Valley, CA 93924 (ISSN 0030-8730) publishes 5 volumes per year. Application to mail at Second-class postage rates is pending at Carmel Valley, California, and additional mailing offices. Postmaster: send address changes to Pacific Journal of Mathematics, P.O. Box 969, Carmel Valley, CA 93924.

\section{PUBLISHED BY PACIFIC JOURNAL OF MATHEMATICS, A NON-PROFIT CORPORATION}




\section{Pacific Journal of Mathematics}

Vol. 138, No. $2 \quad$ April, 1989

Bruce Alan Barnes, Operators which satisfy polynomial growth

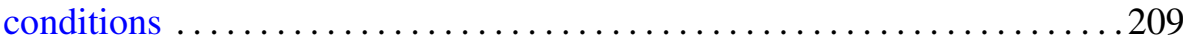

Shiferaw Berhanu, Propagation of hypo-analyticity along

bicharacteristics

Christopher J. Bishop, Lennart Carleson, John Brady Garnett and

Peter Wilcox Jones, Harmonic measures supported on curves . . . . . . 2233

Chong-Man Cho, $M$-ideals of compact operators

Kenneth R. Davidson and Domingo Antonio Herrero, Quasisimilarity of nests

Fumio Hiai and Yoshihiro Nakamura, Distance between unitary orbits in

von Neumann algebras ................................ 259

Krzysztof Jarosz, Small isomorphisms of $C(X, E)$ spaces $\ldots \ldots \ldots \ldots \ldots 295$

Robert Kusner, Comparison surfaces for the Willmore problem ..........317

Nativi Viana Pereira Bertolo, On the Hardy space $H^{1}$ on products of

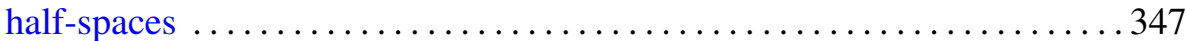

Max Shiffman, Measure-theoretic properties of nonmeasurable sets

Sechiko Takahashi, Extension of the theorems of

Carathéodory-Toeplitz-Schur and Pick 